Research Paper

\title{
Dissection of Targeting Molecular Mechanisms of Aristolochic Acid-induced Nephrotoxicity via a Combined Deconvolution Strategy of Chemoproteomics and Metabolomics
}

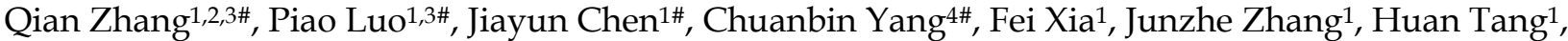 \\ Dandan Liu ${ }^{1}$, Liwei $\mathrm{Gu}^{1}$, Qiaoli Shi ${ }^{1}$, Xueling $\mathrm{He}^{1}$, Tong Yang ${ }^{1}$, Jigang Wang ${ }^{1,2,3,4,5,6,7 凶}$ \\ 1. Artemisinin research center, and Institute of Chinese Materia Medica, China Academy of Chinese Medical Sciences, Beijing 100700, China. \\ 2. School of Chinese Materia Medica, and State Key Laboratory of Component-based Chinese Medicine, Tianjin University of Traditional Chinese Medicine, Tianjin \\ 301617, China. \\ 3. School of Traditional Chinese Medicine, Southern Medical University, Guangzhou 510515, China. \\ 4. Department of Urology, The Second Clinical Medical College, Jinan University (Shenzhen People's Hospital), Shenzhen, Guangdong 518020, China. \\ Center for Reproductive Medicine, Dongguan Maternal and Child Health Care Hospital, Southern Medical University, Dongguan 523125, China. \\ 6. Central People's Hospital of Zhanjiang, Zhanjiang, Guangdong 524037, China. \\ 7. Guangdong Provincial Key Laboratory of New Drug Screening, School of Pharmaceutical Sciences, Southern Medical University, Guangzhou 510515, China. \\ \#These authors contributed equally to this work. \\ $\bowtie$ Corresponding author: Tel: +86 10 64096302; Fax: +86 10 64096302; E-mail: jgwang@icmm.ac.cn (Jigang Wang). \\ () The author(s). This is an open access article distributed under the terms of the Creative Commons Attribution License (https://creativecommons.org/licenses/by/4.0/). \\ See http://ivyspring.com/terms for full terms and conditions.
}

Received: 2021.11.30; Accepted: 2022.01.11; Published: 2022.02.21

\begin{abstract}
Aristolochic acid (AA), mainly derived from herbal Aristolochia and Asarum plants, was listed as a human carcinogen class I in 2002. Aristolochic acid nephropathy (AAN) is a rapidly progressive tubulointerstitial nephritis and urothelial cancer caused by AA. However, the targeting molecular mechanisms of AAs-induced nephrotoxicity are largely unclear. This study aims to dissect targeting molecular mechanisms of AA-induced nephrotoxicity. Activity-based protein profiling (ABPP) in combination with cellular thermal shift assay (CETSA) was performed to identify the AAs binding target proteins. Our data indicated that several key enzymes in the metabolic process and mitochondrial respiration including IDH2 and MDH2 (Krebs cycle), PKM and LDH (aerobic respiration), FASN (fatty acid beta-oxidation), HK2 (glucose metabolism), and ATP synthase were identified as directly binding targets of AAs. Metabolomics and oxygen consumption rate (OCR) experiments further confirmed that AAs targeting proteins disrupted metabolic biosynthesis processes and impaired mitochondrial functions. Ultimately, AAs induced renal cells apoptosis by disturbing various biological processes. Cumulatively, AAs may directly bind to key proteins involved in the metabolic process and mitochondrial homeostasis, and finally induce aristolochic acid nephropathy. Our findings provide novel insight into underlying mechanisms of AAs-induced kidney toxicity, which may help to develop therapeutic strategies for AAN.
\end{abstract}

Key words: Aristolochic acid nephropathy, chemical proteomics, mitochondrial dysfunction, apoptosis, metabolism

\section{Introduction}

Aristolochic acid nephropathy (AAN), previously known as Chinese herb nephropathy $(\mathrm{CHN})$, is a rapidly progressive tubulointerstitial nephritis causing end-stage renal disease and urothelial carcinoma [1]. This nephropathy is mainly caused by aristolochic acids (mainly aristolochic acid I and aristolochic acid II) (AAs) [2,3]. AAs are a class of natural products mainly derived from herbal medicinal plants, such as Aristolochia and Asarum [4, 5]. Epidemiological investigations indicated that people in the Balkans accidentally ingested AAcontaminated wheat and groundwater or mistakenly used it as a weight-loss drug, leading to Balkan endemic nephropathy (BEN) in 1990s [6-8]. Later this similar etiology of renal disease and the upper tract urothelial cancer (UTUC) induced by AAs have 
emerged worldwide (mainly in East Asia and Southeast Asia) [9-11], which related to the fact that people in these areas are accustomed to consuming traditional Chinese medicine (TCM). Thus, the toxic effects induced by AAs have become a serious global public health problem. Due to the toxicity, AA was listed as a human carcinogen class I by the World Health Organization International Agency for Research on Cancer in 2002.

AAs preferentially target proximal tubular epithelial cells (PTEC) to induce nephrotoxicity [12]. Nephrotoxicity caused by AAs has multiple mechanisms, including immune-inflammation, apoptosis, oxidative stress, hemodynamic abnormalities and endoplasmic reticulum stress [13-17]. For instance, it was reported that AAs metabolites, aristolactam-nitrenium ions, react with DNA bases to form the covalent AA-DNA adducts [18, 19], thereby leading to nephrotoxic, genotoxic, and carcinogenic effects. However, the direct binding targets and exact molecular mechanisms of AA-induced nephrotoxicity are remaining unclear.

Chemical proteomics is a novel and promising approach that can systematically identify the covalent binding targets of compounds [20,21]. Previously, we utilized this approach to identify the underlying targeting mechanisms of many natural products, such as artemisinin, celastrol, curcumin and andrographolide [20-23]. This contributes to further understanding the potential mechanisms of pharmacology and toxicology of natural products.

Here, a chemical proteomics method based on the activity-based protein profiling (ABPP) was used to comprehensively profile and identify the AAs' covalent binding targets in vivo and in vitro. A wealth of protein targets is involved in multiple important biological processes, especially mitochondrial metabolism and cellular respiration. Among them, several key enzymes were validated by cellular thermal shift assay (CETSA) and pull-down western blot experiments. Interestingly, the activities of target enzymes were impaired by AAs. Therefore, these results suggest that AAs may induce nephrotoxicity through a complex protein-targeting mechanism. In addition, we further validated the AAs targets involved in metabolic processes and mitochondrial dysfunction by using metabolomics, oxygen consumption rate (OCR) and mitochondrial membrane potential (MMP) assays. Our data support a promising strategy to better understand the molecular mechanisms of toxic compounds.

\section{Results}

\section{Synthesis and bioactivity of aristolochic acid probe}

To identify AAs direct binding proteins using ABPP, two aristolochic acid probes (AP1, AP2) conjugated with a clickable alkyne tag respectively were chemically designed and synthesized (Figure 1A). The design and synthesis schemes of two AA-probes are shown in Scheme 1. We first compared the cell toxicity effect of AA-probes and AAs by using human renal proximal tubule epithelial cells (HK-2 cells). Our results showed that the toxicity effect of AP2 was as potent as the original compound, aristolochic acid I (AAI) sodium salt, while AP1 displayed strong toxic effects (Figure 1B). However,
(1)<smiles>[R]c1cc(OC)c2cc([N+](=O)[O-])c3c(C(=O)O)cc4c(c3c2c1)OCO4</smiles>

Aristolochic acid I: $\mathrm{R}=\mathrm{H}$

Aristolochic acid IVa: $\mathrm{R}=\mathrm{OH}$
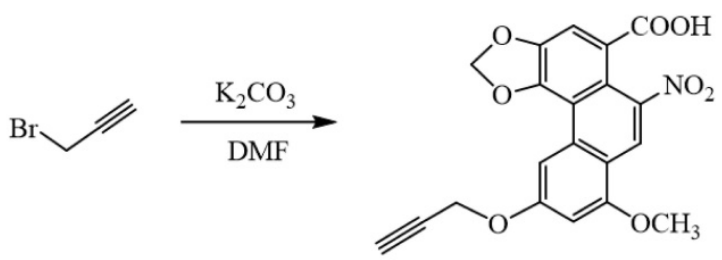

Aristolochic acid I probe (AP1)

(2)<smiles>[R]c1ccc2cc([N+](=O)[O-])c(C(=O)[O-])cc2c1</smiles><smiles>C#CCBr</smiles>

Aristolochic acid II: $\mathrm{R}=\mathrm{H}$

Aristolochic acid IIIa: $\mathrm{R}=\mathrm{OH}$

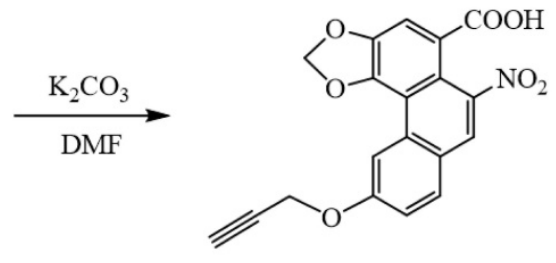

Aristolochic acid II probe (AP2)

Scheme 1. Procedures for the preparation of aristolochic acid probes. (1) Chemical structures of aristolochic acid I (AAI), Aristolochic acid IVa (AAIVa) and the alkyne-tagged-clickable probe (aristolochic acid I probe, AP1). (2) Chemical structures of aristolochic acid II (AAIl), Aristolochic acid IIla (AAllla) and the alkyne-tagged-clickable probe (aristolochic acid II probe, AP2). 
the toxicity effect of two analogues (AAIVa, AAIIIa) was significantly lower than AAI (Figure S1A). Collectively, the probe AP2 has a similar toxicity profile to AAI sodium salt. Thus, AP2 was selected for identified AAs' binding targets.

\section{Fluorescence labeling of aristolochic acid probe}

To explore the cellular localization of AA-probe in HK-2 cells, live HK-2 cells were treated with AP2 probe for $4 \mathrm{~h}$, washed with PBS, fixed with $4 \%$ paraformaldehyde and permeabilized with $0.2 \%$ Triton-100. Cells were then clicked with fluorescence dye and processed to image. Fluorescent signaling was scattered throughout the cell, indicating that AA-probe distributed in the nucleus and cytoplasm (Figure 1D), suggesting that AA-probe can bind to cell contents (nucleic acid or protein and so on). To a certain extent, this result is similar to previous findings that is the covalent formation of adducts with AAs [18]. Next, to identify and profile the target proteins of AAs, an ABPP assay was carried out in vitro, ex vivo and in vivo. A common workflow of this ABPP approach $[20,21]$ is illustrated in Figure 1C.

Firstly, we optimally chose AA-probe (AP2) for further in-depth experiments by combining the toxicity effects of two probes on HK-2 cells and the results of pre-labeling target proteins experiment in situ. HK-2 cells were incubated with AA-probe and then crude proteins were extracted. Protein samples were processed by click chemistry and separated by SDS-PAGE gel electrophoresis. AA-probe binding to proteins could be visualized by a fluorescence imaging scanner. Many proteins were labeled by AP2 in a dose-dependent manner (Figure 1E). Furthermore, AP2 could bind to different targets that were competed away by pretreatment with AAI (Figure 1F). We also implemented cellular imaging
A<smiles>[R]c1cc([N+](=O)[O-])cc2c(C(=O)O)cc3c(c12)OCO3</smiles>

Aristolochic acid I (AAI) $\left(\mathrm{R}=\mathrm{OCH}_{3}\right)$

Aristolochic acid II (AAII) $(\mathrm{R}=\mathrm{H})$

C

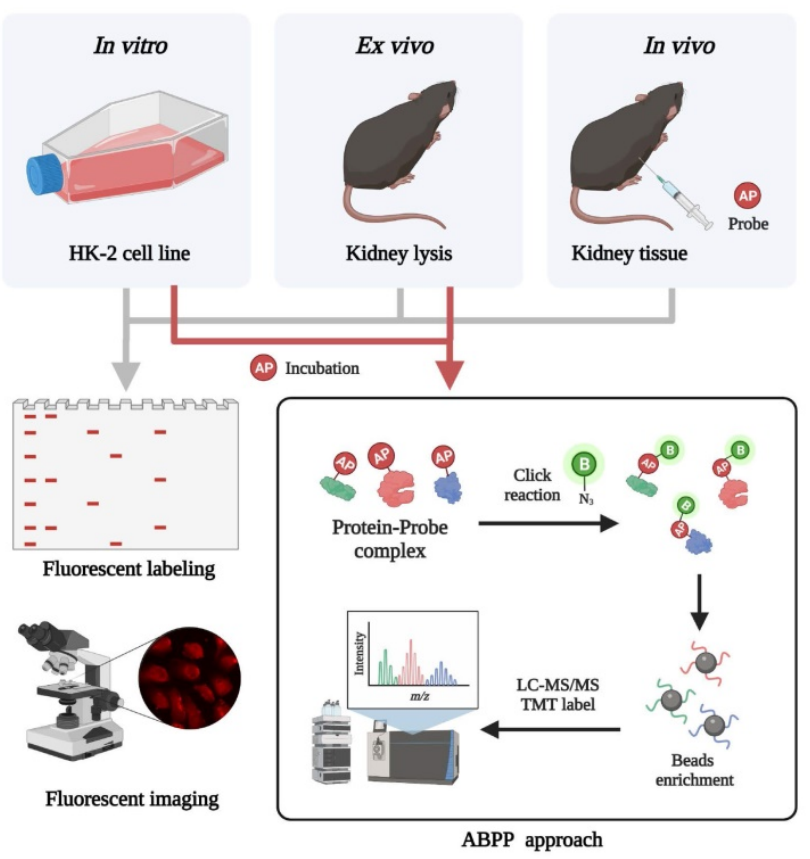

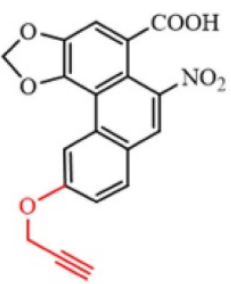

Aristolochic acid II probe (AP2)

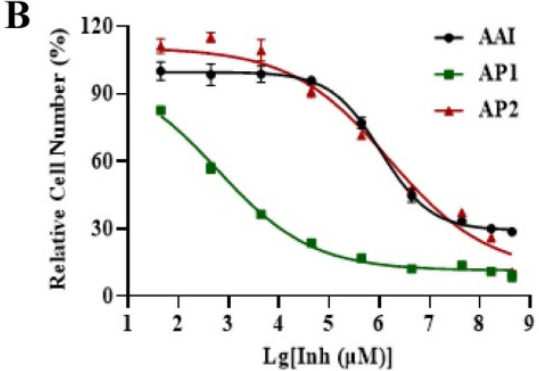

D
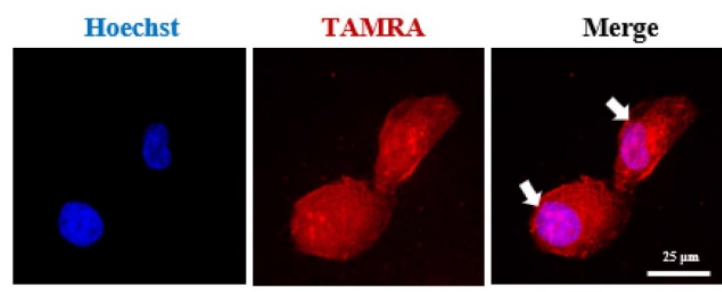

$\mathbf{E}$

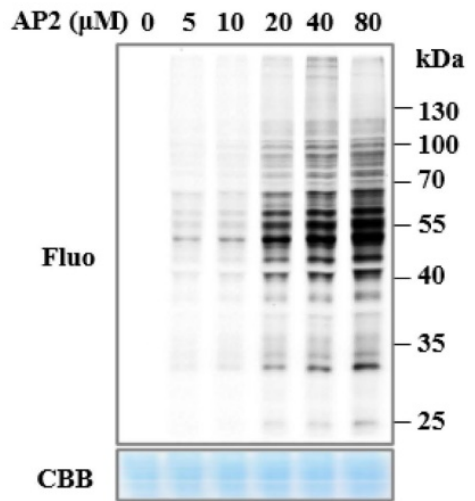

F

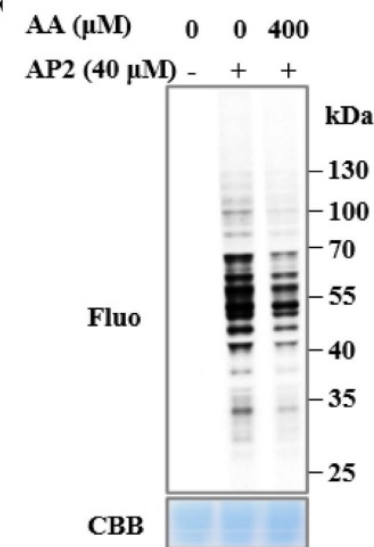

Figure 1. Bioactivity and fluorescence labeling of aristolochic acid probe. (A) Chemical structures of aristolochic acid I (AAI), aristolochic acid II (AAll), aristolochic acid probe 1 (AP1) and aristolochic acid probe 2 (AP2). (B) The toxicity effects of aristolochic acid probes (AP1, AP2) compared with aristolochic acid I (AAll) on HK-2 cell. (C) Schematic model showing the workflow of ABPP approach combining with bio-orthogonal click chemistry. (D) Fluorescent cellular imaging to track the subcellular location of the AP2 $(40 \mu \mathrm{M})$ in HK-2 cells (scale bar $=25 \mu \mathrm{m})$. (E) In situ labeling protein in an AP2 dose-dependent manner in HK-2 cells. (F) The competition of labeling protein with AP2 by excess AA in HK-2 cells. 
fluorescence experiments in situ. Cellular fluorescence intensity varied in an AP2 dose-dependent manner (Figure S1B). Co-incubation with excess AAI could effectively compete with the labeling proteins of AP2 in situ (Figure S1C). Secondly, we conducted experiments similar to the above cellular experiment ex vivo. Proteins were extracted from the kidney of mice and then treated with AP2 via a click reaction. Kidney proteins were labeled by AP2 in a dose-dependent manner and could be competed away by an excess amount of AAI (Figure S1D-E). Moreover, similar cell labelling experiments were also carried out in vivo. Following tail vein injection of AP2, mouse kidneys were collected and extracted proteins were then subjected to click fluorescent labeling. Results showed that AP2 could also bind to many proteins in vivo (Figure S1F). In summary, these results indicate that the AP2 covalent binding targets are similar to aristolochic acid analogues.

\section{Identification of the nephrotoxic targets of aristolochic acid}

Next, to identify the potential binding proteins of AA by ABPP approach, HK-2 cells and mouse kidney lysates were first incubated with AP2 for $4 \mathrm{~h}$. Subsequently, proteins lysates were incubated with biotin-alkyne, and the AP2 binding targets were affinity-purified with streptavidin beads. Samples were digested to peptides and identified by LC-MS/MS according to our previous protocols [20]. Compared to the DMSO group, a total of 898 target proteins were identified as direct binding targets of AAs in HK-2 cells (Figure 2A-B). Meanwhile, a total of 860 human homologous proteins were identified as direct binding targets of AAs in the lysate of mouse kidneys (Figure S2). Among these proteins, 312 proteins were identified both in HK-2 cells and mouse kidney (Figure 2C). Gene ontology (GO) analysis for these 312 shared targets indicated that these proteins participate in key biological processes, including tricarboxylic acid (TCA) cycle, aerobic respiration, fatty acid beta-oxidation, nucleotide metabolic process and purine nucleotide metabolic process (Figure 2D). Interestingly, these metabolism-related target proteins were mainly located on the mitochondria or cytosol (Figure 2E). Thus, AAs may induce nephrotoxicity via direct targeting mitochondrial proteins to affect key metabolic processes such as TCA cycle (Krebs cycle), aerobic respiration, and cellular respiration.

\section{Validation of the nephrotoxic targets of aristolochic acid}

We next conducted protein and protein interaction (PPI) analysis for AA targets involved in key metabolic processes TCA cycle and respiration processes (Figure 3A), as indicated in the GO analysis. Of interest, several rate-limiting enzymes occupied an important position in PPI analysis, such as isocitrate dehydrogenase 2 (IDH2), malate dehydrogenase 2 (MDH2), ATP synthase, pyruvate kinase M1/2 (PKM), and lactate dehydrogenase A/B (LDH). Therefore, these targets were selected for verification of their bind with AP2 in vitro. Since these enzymes are mainly distributed in mitochondria. Here, we first used a red fluorescence dye of AA-probe (AP2) to track its cellular co-localization with mito-tracker green, a mitochondrion-selective. As expected, the fluorescence imaging confirmed the co-localization of AP2 with mitochondria in HK-2 cells (Figure 3B). Thus, AAs may target multiple mitochondria proteins.

A pull-down experiment was then used to validate the AP2 binding to these targets in the kidney lysates. The results indicated that AP2 effectively pulled down IDH2, MDH2, pyruvate carboxylase (PC), voltage-dependent anion-selective channel protein 1 (VDAC1), PKM and LDHA (Figure 3C). Meanwhile, the binding of AP2 to these targets could be competed against by AAs (Figure 3C), further suggesting that these proteins may be direct targets of AAs. We also validated that AAs targeted several selected metabolic enzymes and mitochondria-related proteins by pull-down experiment, which include acetyl-CoA carboxylase 1 (ACACA), ATP synthase, fatty acid synthase (FASN) and hexokinase-2 (HK2) (Figure S3A). The CETSA based on the thermodynamic stabilization of protein is regarded as an effective approach for monitoring the compound binding to protein [24]. CETSA-WB experiment in kidney lysate was also performed to validate the direct binding proteins to AAs. Our results showed that IDH2, MDH2, PKM, LDHA, HK2, VDAC1 and PC all occupied more significant thermal stabilization in the AAI treatment group (Figure 3D, Figure S3B). Moreover, the immunofluorescence staining confirmed the co-localization of AP2 with IDH2, MDH2, ATP synthase or PKM in HK-2 cells (Figure $3 \mathrm{E}$, Figure S3C). To verify the interaction of AAI-target proteins, we carried out molecular docking analysis on the solved structures of HK2, PKM, LDHA (glucose metabolism), ATP synthase, IDH2, MDH2 (TCA cycle), FASN (fatty acid metabolism) and VDAC1 proteins. The analysis results suggested the interactions between AAI and these representative target proteins (Figure S4A-H, supporting information). 
A

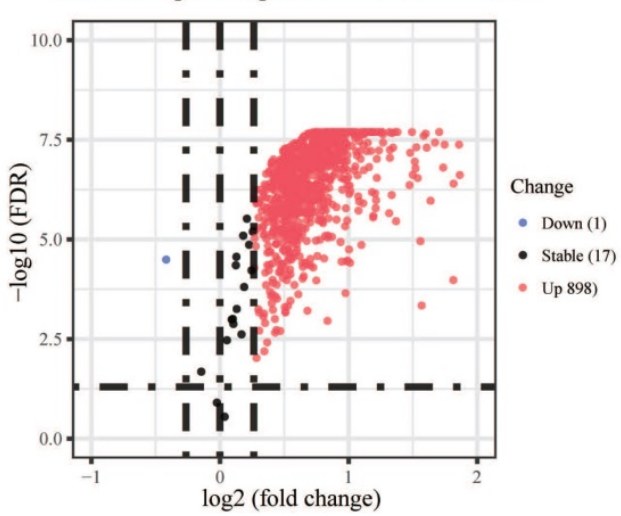

B

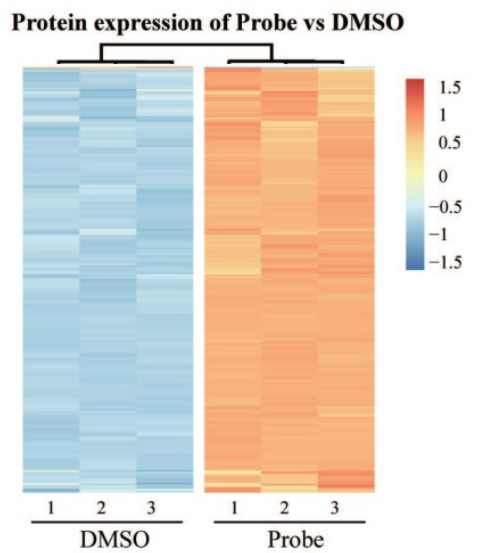

C Overlap protein detected in different groups

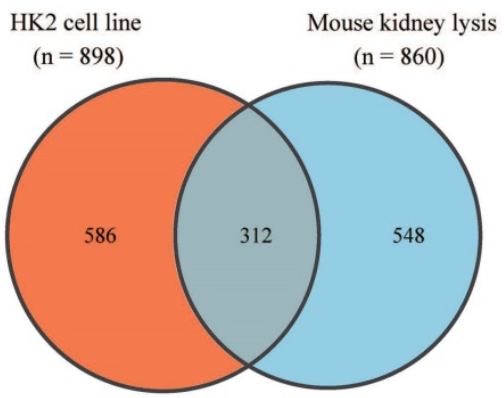

D

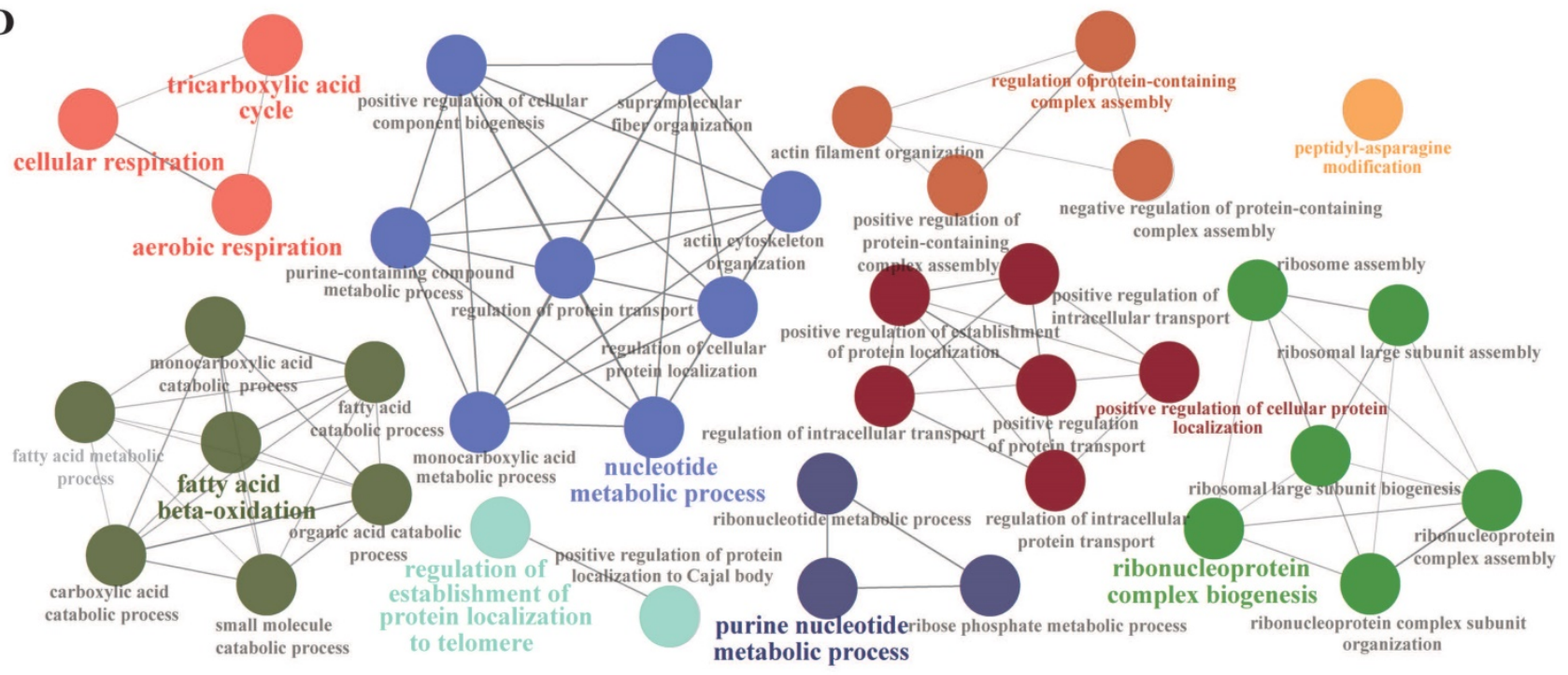

E

\begin{tabular}{|c|c|c|}
\hline Target proteins & Subcellular location & Molecular functions and biological processes \\
\hline IDH2 & Mitochondria & Tricarboxylic acid (TCA) cycle, cellular respiration \\
\hline MDH2 & Mitochondria & Tricarboxylic acid (TCA) cycle, cellular respiration, glycolysis \\
\hline ATP synthase & Mitochondria & Cellular respiration, electron transport complexes of the respiratory chain \\
\hline PKM & Cytosol & Aerobic respiration, glycolysis, pyruvate metabolism \\
\hline LDH & Cytosol & Aerobic respiration, glycolysis, lactic acid metabolism \\
\hline IDH2, isocitrate dehydrogenase (NADP(+)) 2; MDH2, malate dehydrogenase 2; ATP5F, ATP synthase F1; PKM, pyruvate kinase; M1/2; LDH, lactate dehydrogenase A/B
\end{tabular}

Figure 2. Aristolochic acid targeting proteins participate in key metabolic processes in kidney. (A) Volcano plot depicting the differential enrichment of proteins in AP2 (probe) vs DMSO groups. (B) Temporal patterns for AA targeting proteins (AP2 vs DMSO). (C) Venn digraph depicting the same target proteins of AA in kidney tissues and HK-2 cells. (D) Target proteins involved in essential biological processes by Gene Ontology (GO) analysis. (E) The representative AA targets and their biological functions.

In addition, western blot assays indicated that the expressions of IDH2, MDH2, HK2, VDAC1 and PC were down-regulated in AA-treated kidneys, but not LDHA compared with DMSO group (Figure 3F), further supporting the notion that AAs affect key metabolic processes. ATP synthase and PKM are crucial enzymes involved in glucose metabolism, ATP production and cellular respiration. Enzyme activity assays showed that AAs inhibit their enzyme catalytic activities (Figure 3G-H). Collectively, we systematically profiled and identified the targets of AAs in HK-2 cells and kidneys by a novel ABPP approach combining with CETSA, revealing that AAs may cause nephrotoxicity via targeting multiple key metabolic enzymes. 
A

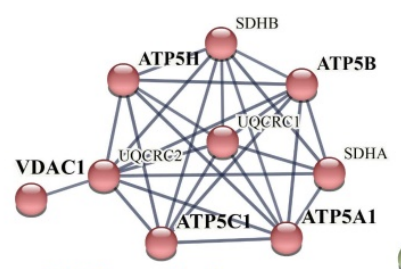

Cellular respiration

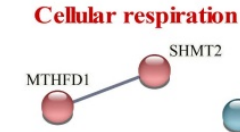

Aerobic respiration, glycolys

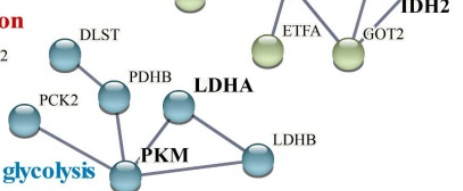

B

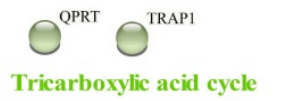

Tricarboxylic acid cycle
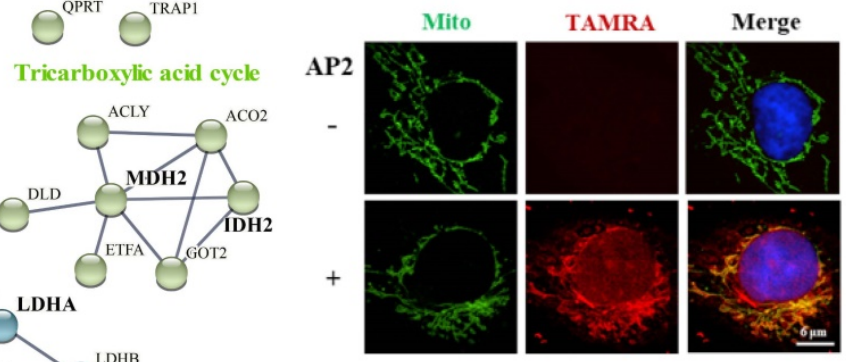

C

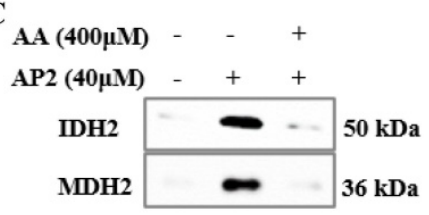

AA $(400 \mu M)$

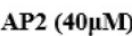

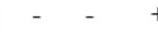

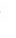

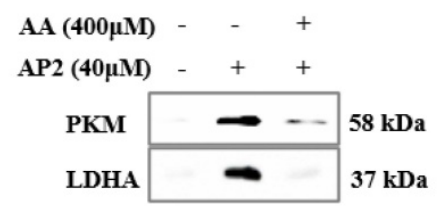

D
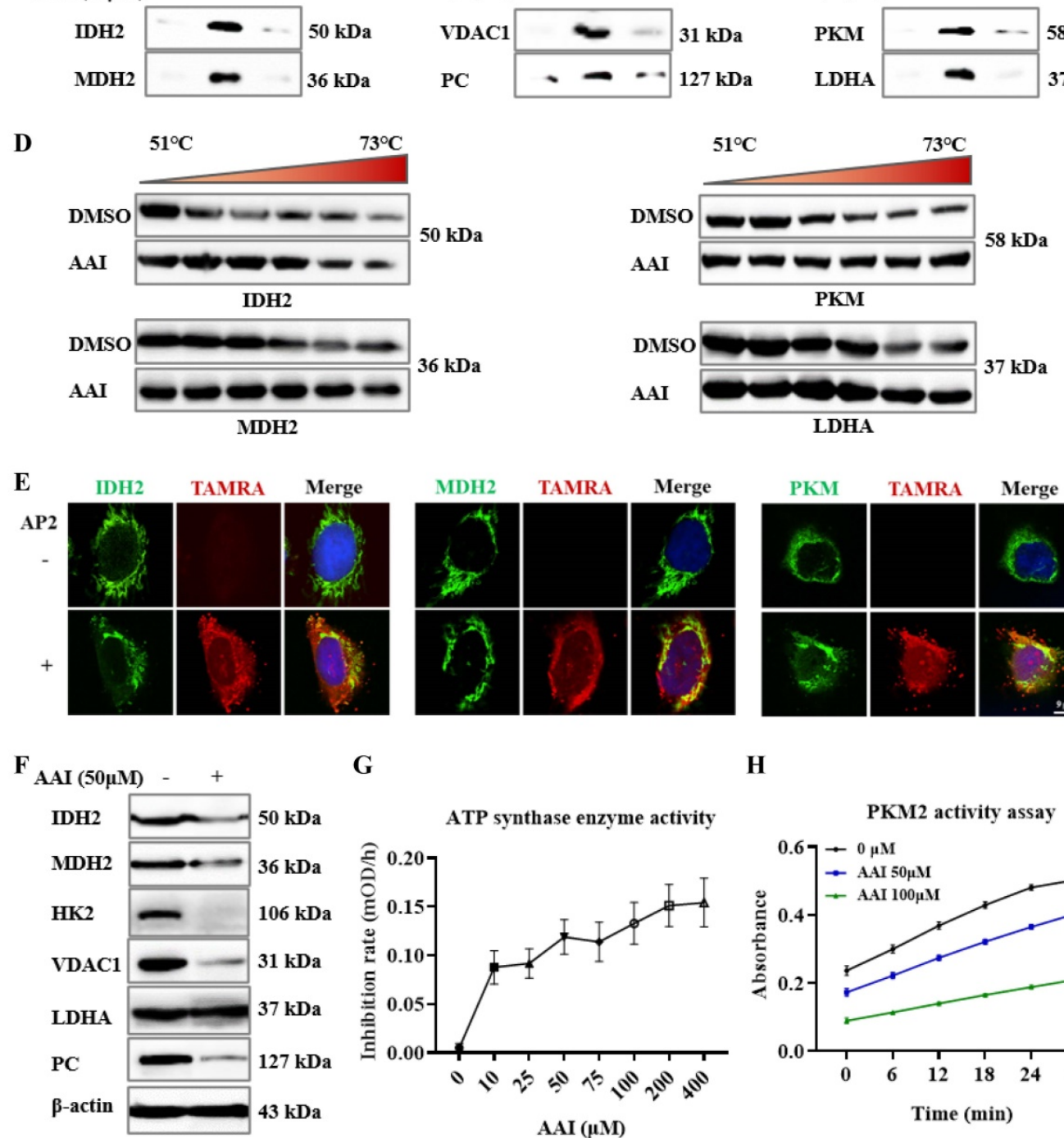

G
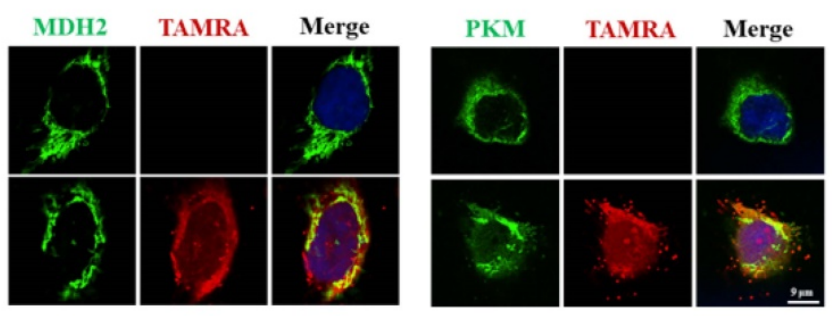

H
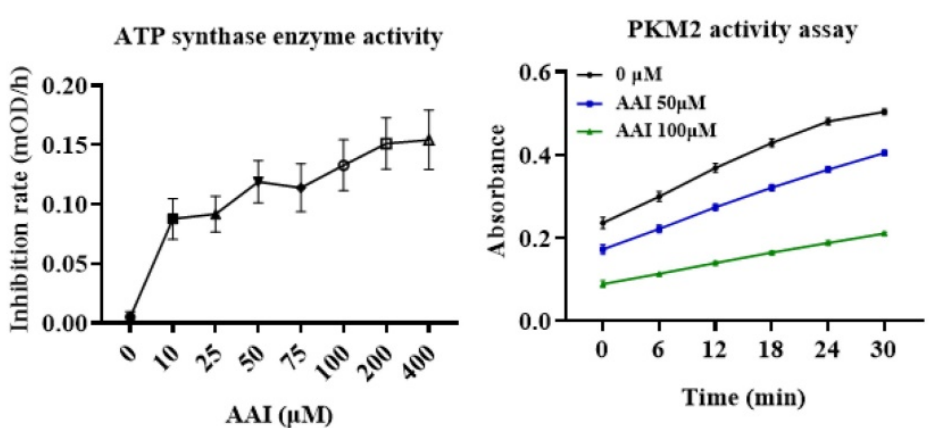

Figure 3. Aristolochic acid targets multiple mitochondrial proteins and metabolic enzymes. (A) The PPI analysis of AA targets involved in tricarboxylic acid (TCA) cycle and respiration processes. (B) Fluorescence co-localization of AA-probe (a red fluorescence dye) with mitochondria (green) in HK-2 cells by using confocal microscopy to image, scale bar $=6 \mu \mathrm{m}$. (C) Pull-down western blotting study to verify AA directly targeting IDH2, MDH2, VDAC1, PC, PKM and LDHA proteins. (D) CETSA-WB experiment to validate the $A A$ binding to IDH2, MDH2, PKM and LDHA proteins. (E) Fluorescence staining of target proteins (green) and AA-probe (a red fluorescence dye), scale bar $=9$ $\mu \mathrm{m}$. (F) Expression of IDH2, MDH2, HK2, VDAC1, PC and LDHA proteins in AA treatment or DMSO group. (G) The ATP synthase enzyme activity with or without AAs in the lysate of kidney. (H) The PKM2 activity of rhPKM2 with or without AAs.

\section{Metabolomic analysis of aristolochic acid in kidney and serum}

To further interrogate the impairment of metabolic processes after exposure to AAs in vivo, AA-induced nephrotoxicity mouse model was established by intraperitoneal injection with AAI. The basic parameters of the animal were monitored to 
evaluate whether AAI caused kidney injury in our study (Figure 4A). Compared to the control group, AAI not only significantly reduced body weight, but also resulted in an increase of the kidney/body weight ratio (Figure 4B, Figure S5B). AAI caused kidney injury and morphological changes (Figure $4 \mathrm{C}$, Figure S5A, Figure S5B), as evidenced by aggravating hemorrhagic necrosis across the cortex and tubules in H\&E staining images. AAI also disordered changes in biochemical parameters, such as UREA and creatinine (CRE) (Figure 4D). Routine blood tests showed that the treatment group had signs of body weakness (Figure S5C). These results indicated AAI-induced nephrotoxic effects in mice.

A nontargeted metabolomics method in kidney tissues and serum of DMSO and AAI-treated mice was further used to characterize AAI-induced toxicity. A total of 1387 metabolites were detected in both positive and negative ion modes by UHPLCMS/MS technique. The pearson correlation coefficient was analyzed between quality control (QC) samples. The higher correlation of QC kidney tissues (the $\mathrm{R}^{2}$ value close to 1 ) showed that the stability and quality of the whole detection process was better in both positive or negative ion modes (Figure S6A-B). Next, all metabolites combined with both positive and negative ion modes for the kidney tissues were analyzed by principal component analysis (PCA), indicating that there were significant differences in the DMSO and AAI-treatment group (Figure 4E). Orthogonal partial least squares-discriminant analysis (Ortho PLS-DA) also showed that metabolites were the separation between the DMSO and treatment group (Figure $4 \mathrm{~F}$ ). Altogether, these results suggest that the classification model is stable and reliable.

Next, differential metabolites in kidneys after AAI-treatment were identified and shown in in a heatmap (Figure 4G). Volcano map depictions were used to visually display the overall distribution of different metabolites (Figure $4 \mathrm{H}$ ). The abscissa represented the change in the fold change of metabolites in different groups ( $\log _{2}$ FoldChange), and the ordinate represented the significance level of difference (-log10 p-value). Each point in the volcano map represents a metabolite. The 287 significantly up-regulated metabolites were represented by red dots, the 352 significantly down-regulated metabolites were represented by blue dots, and the size of the dot represented the VIP value. In addition, we noted metabolites by human metabolome database (HMDB) classification. Results showed that changed metabolites after AAI-treatment were mainly organic acids and derivatives, lipids and lipid-like molecules, and organoheterocyclic compounds (Figure 4I). Subsequently, we performed KEGG enrichment annotation analysis on the differential metabolites, suggesting that AAI may affect purine metabolism, arginine biosynthesis, amino acids metabolism and TCA cycle in kidney (Figure 4J). Moreover, metabolomics studies in serum of mice after AAI-treatment were also analyzed and showed similar results to the kidney tissue as mentioned above (Figure S6C-I). Overall, metabolomic results in the kidney tissues and the serum indicated that the AAI impairs key metabolic pathways, which is consistent with our ABPP results as mentioned above.

\section{Metabolic dysfunction caused by aristolochic acid via inducing mitochondrial damage}

Mitochondria is a central organelle for cellular metabolism and homeostasis, and mitochondrial dysfunction is closely associated with metabolic diseases and drug-induced organ injury [25, 26]. Mitochondrial injuries had been found in AAN patients and animal models of AAN [27, 28]. MMP is considered to be one of the most crucial indicators for evaluating mitochondrial damage. Here, we utilized the JC-1 detection kit to monitor MMP after treatment with AAI. The red/green fluorescence intensity ratio significantly decreased via JC-1 staining (Figure 5A-B), suggesting that AAI induced abnormalization of MMP in HK-2 cells.

To further evaluate the effects of AAI and AAIIIa on the mitochondrial functions, we used an OCR experiment to detect mitochondrial function and cell metabolism in HK-2 cells. OCR is mainly caused by mitochondrial electron transfer and is commonly used to assess the mitochondrial oxidative phosphorylation function. Our results showed that both AAI and AAIIIa effectively reduced basal and maximal respiration of HK-2 cells in a dose-dependent manner, while ATP production, spare respiratory capacity and non-mitochondrial oxygen consumption of HK-2 cells were significantly decreased after AAI and AAIIIa treatment (Figure 5C-D, Figure S7A-C). Collectively, AAI and its analogue AAIIIa induced mitochondrial dysfunction, thereby inhibiting cellular respiration and metabolism.

Mitochondrial dysfunction affects cell energy metabolism, and even induces cell apoptosis. We thus measured several indicators related with mitochondrial apoptosis by western blotting assay. AAI remarkably up-regulated the expression of Bax and down-regulated the expression of Bcl-2 (Figure 5E). AAI increased the release of cytochrome C (Cyt C) in the cytoplasm, suggesting that AAI could cause changes in mitochondrial membrane permeability and loss of transmembrane potential. Subsequently, AAI activated caspase- 3 and caused mitochondrial apoptosis (Figure 5F). In addition, AAI induced 
apoptosis of renal cells by TUNEL staining in AA-treated mice (Figure 5G-H). These results indicated that mitochondrial dysfunction induced by

A
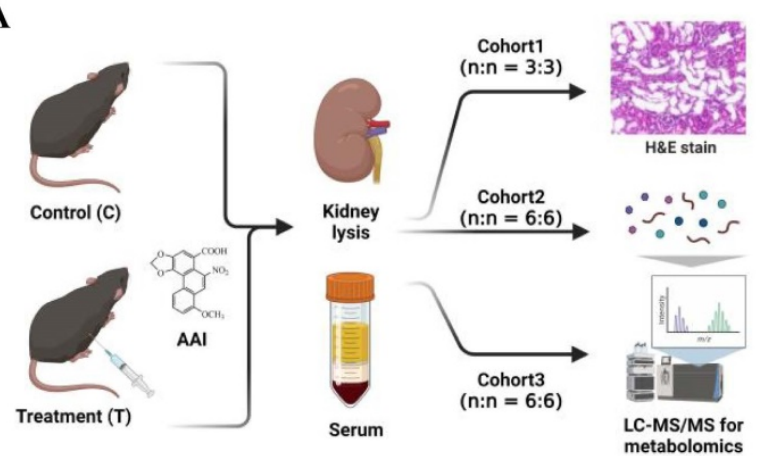

C

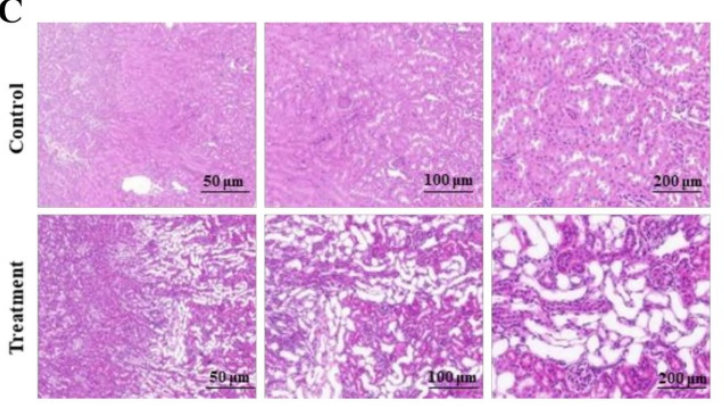

AAI may result in metabolism disorder and mitochondrial apoptosis.

B

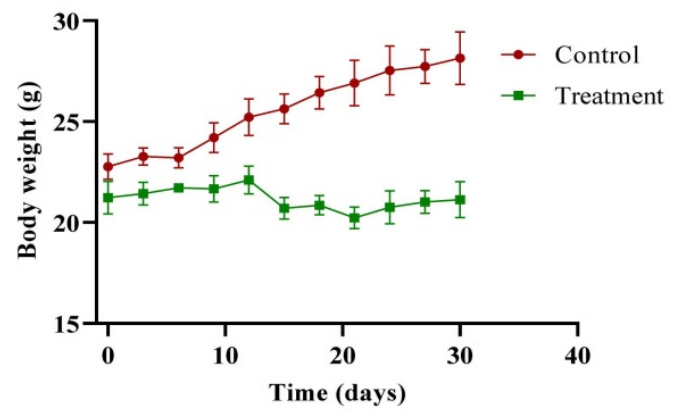

D
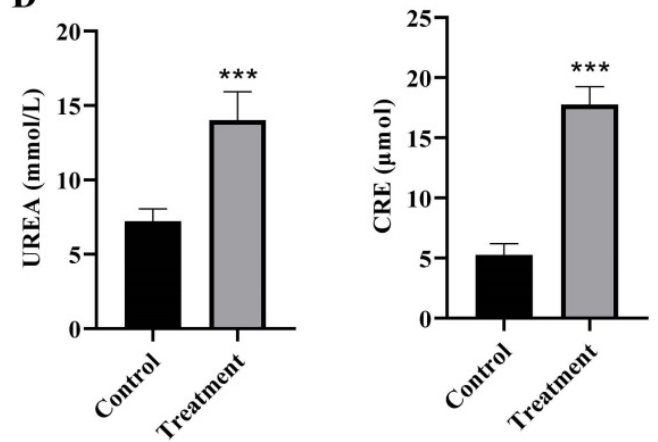

G
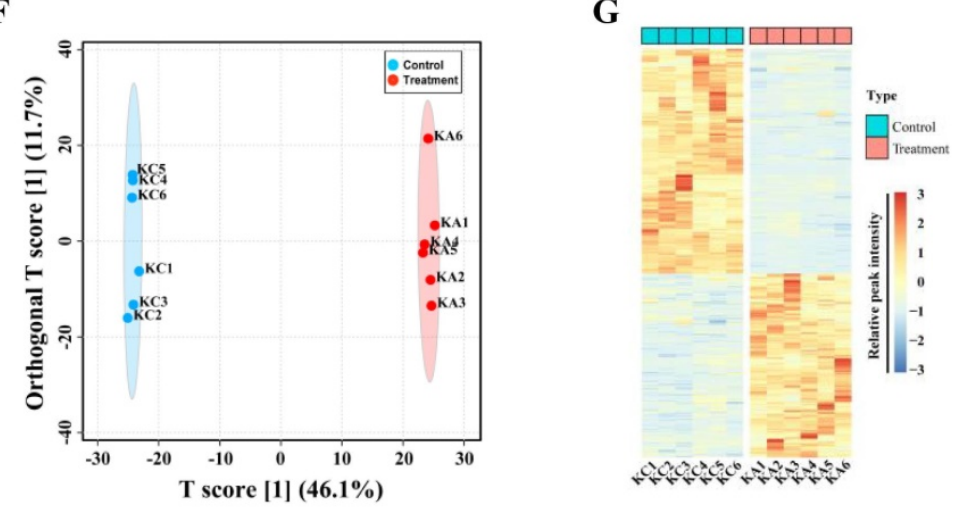

I

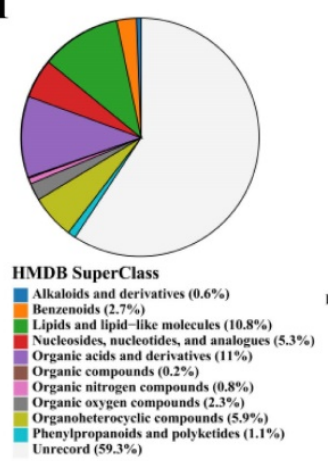

Figure 4. Metabolomics reveals AA-induced nephrotoxicity mainly through purine metabolism, amino acids metabolism and TCA cycle. (A) The strategy for metabolomics on AAl-induced nephrotoxicity. (B) Body weight from AAl-treated (treatment group) and control group mice. (C) Representative H\&E staining in the kidneys of AAl-treated and control mice. (D) Biochemical indicators for UREA and CRE in the serum of mice. (E) The PCA plots of the DMSO and AAl-treatment groups in the kidney, *** $P<0.001$, compared with control $(n=5)$. (F) Ortho PLS-DA analysis of the DMSO and treatment groups in the kidney. (G) Heatmap showing top up and down metabolites in the kidney after AAl-treatment. (H) The volcano map displays the different metabolites in the kidney after AAl-treatment. Up-regulated metabolites were represented by red dots, down-regulated metabolites were represented by blue dots. (I) Classification information of HMDB database annotations in the kidney after AAI-treatment. (J) KEGG biochemical metabolic pathway and signal transduction pathway in the kidney after AAI-treatment. 

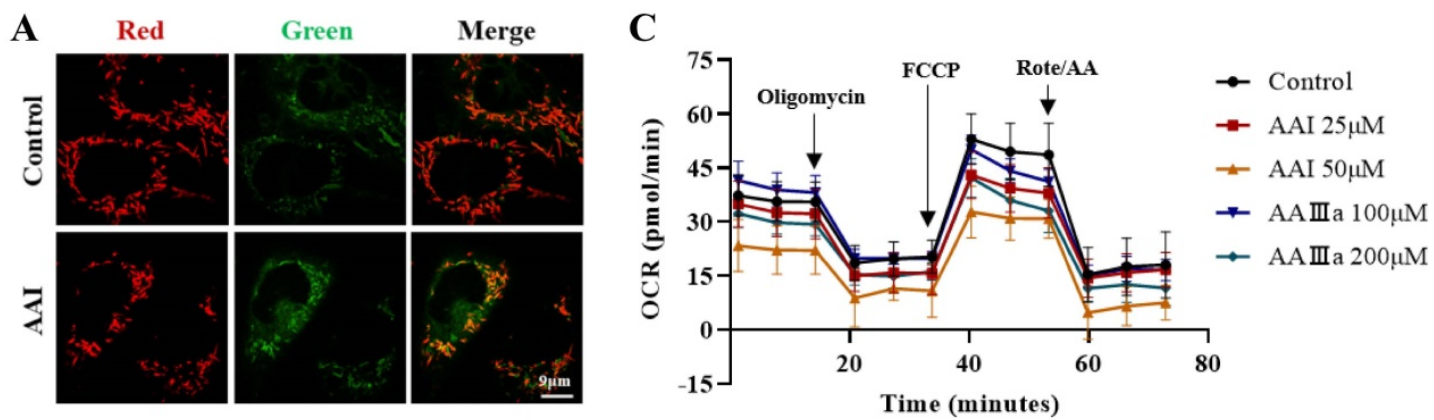

B
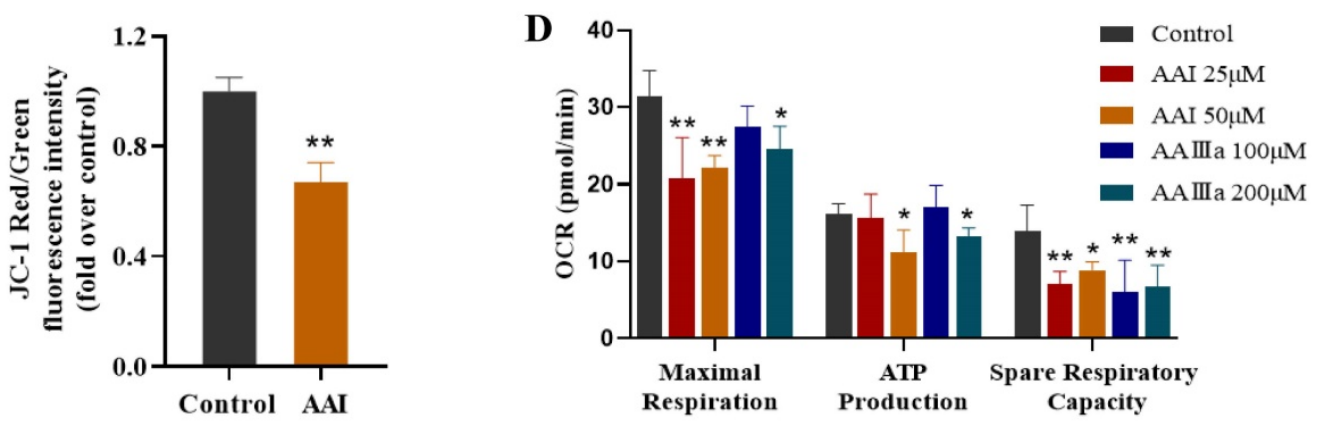

$\mathbf{E}$
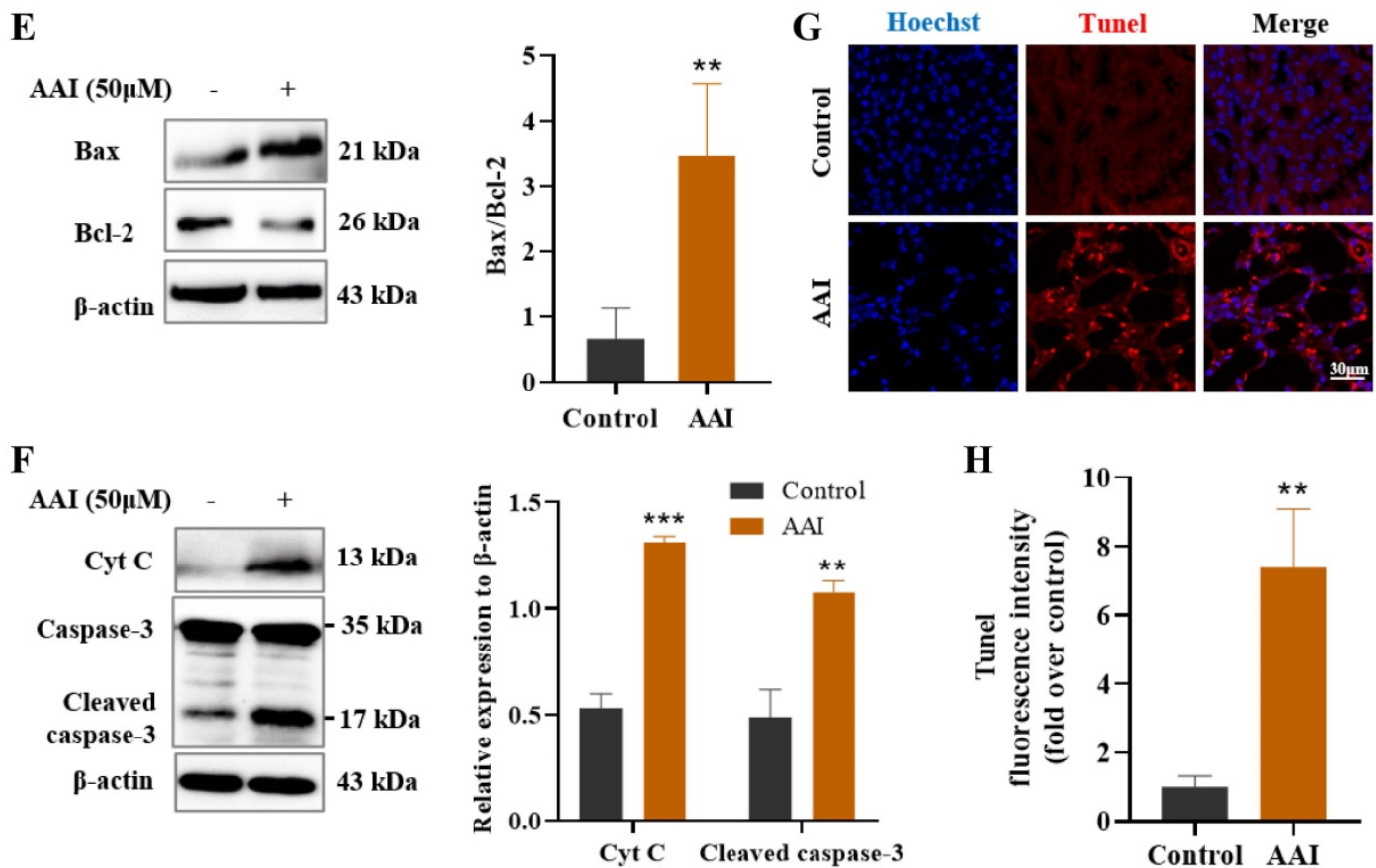

Figure 5. Mitochondrial dysfunction caused by AAl-induced metabolism disorder and apoptosis. (A) The JC-1 staining for mitochondrial membrane potential, scale bar $=9 \mu \mathrm{m}$. (B) Fluorescence intensity quantitative statistics correspond to Figure 5A. (C) The determination of oxygen consumption rate (OCR). (D) The indicators of OCR including maximal respiration, ATP production and spare respiratory capacity. (E) The indicators of mitochondrial apoptosis Bax and Bcl-2 proteins by western blotting and quantitative statistics. (F) The indicators of mitochondrial apoptosis $C y t C$ and caspase-3 proteins by western blotting and quantitative statistics. (G) Tunel staining in the kidneys of mice after AAl treatment, scale bar $=30 \mu \mathrm{m}$. $(\mathbf{H})$ Fluorescence intensity quantitative statistics correspond to Figure $5 \mathrm{G}$. $\mathrm{n}=3, * P<0.05$, $* * P<0.01, * * * P<0.001$ compared with control.

\section{Discussion}

AAs-induced AAN, characterized by interstitial nephritis, tubular atrophy and renal fibrosis, is a major global health problem [29]. However, the direct binding protein targets of AAs in the kidney are missing and AA-induced nephrotoxicity is not fully understood. In this study, we utilized an $A B P P$ combined with CETSA and metabolomic approaches to investigate AAs-binding proteins and characterized AAs-induced nephrotoxicity. Our results indicated that AAs can covalently target many proteins associated with metabolic processes and mitochondrial function, suggesting that the metabolic disorder and mitochondrial dysfunction induced by AAs results in nephrotoxicity (Figure 6). 


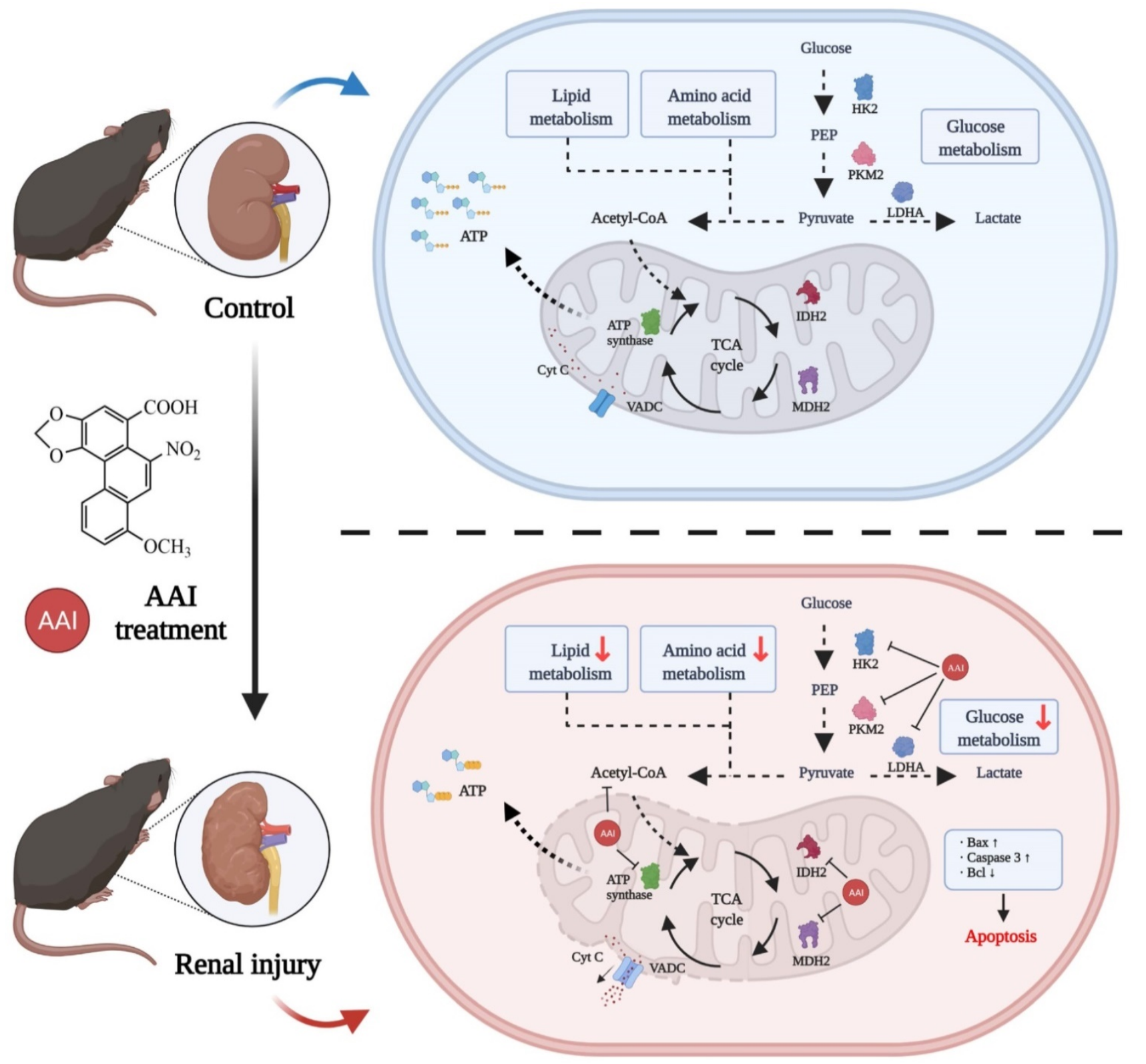

Figure 6. Schematic model showing the molecular mechanisms of the AA-induced nephrotoxicity. Activity-based protein profiling (ABPP) in combination with cellular thermal shift assay (CETSA), as well metabolomic studies showed that AAs directly target multiple key enzymes in the metabolic process such as lipid metabolism, amino acid metabolism, and TCA cycle, and then impairs mitochondrial dysfunction and induces apoptosis. Overall, our study provides novel insight into underlying mechanisms of AAs-induced kidney toxicity and pathogenesis.

Previously, AA-DNA adduct formation has been shown to mediate $\mathrm{A}>\mathrm{T}$ transversions to induce genotoxicity and carcinogenesis [30, 31]. The underlying mechanism is due to aristolochic acid bioactivated to an electrophilic cyclic aristolactamnitrenium ion with delocalized positive charge, covalently binding to the exocyclic purine nucleotides of DNA [18, 32, 33]. Here, we designed aristolochic acid probes, and then identified AA-targeting proteins via the ABPP method. Interestingly, our experimental results showed that the targeting mechanisms of actions of AAs are like an exploding bomb, which impaired multiple crucial metabolic processes and mitochondrial dysfunction and then caused renal cell apoptosis or death.

Recent studies have shown that metabolomics can identify and profile functional metabolites to reveal the varied pathological mechanisms of AAs-induced nephrotoxicity, which including the inhibition of amino acid metabolism, fatty acid metabolism, glucose metabolism and the TCA (Krebs) cycle [34-37]. However, the targeting mechanism of action is unclear. Notably, our results indicated that AAs can directly target to certain key enzymes in the metabolic process including IDH2 and MDH2 (Krebs cycle) [38], PKM and LDH (aerobic respiration) [39], FASN (fatty acid beta-oxidation) [40], HK2 (glucose metabolism) [41] and ATP synthase (cellular 
respiratory chain). Subsequently, AAs targeting several vital enzymes were demonstrated by pulldown and cellular colocalizing image experiments. In addition, CETSA assays further validated that AAs could directly bind to IDH2, MDH2, PKM, PC, LDHA and HK2 proteins. Enzyme activity assays showed that AAs inhibited their enzymatic function and expression. Here, we further validated that AAs exposure results in aberrant metabolic pathways similar to the above approach via a metabolomic method. Although our study is useful for identifying AAs covalent targets, this experimental approach is inadequate for the identification of non-covalent or reversible binding proteins of aristolochic acid. It will be important to determine the function of targeting proteins in AA-induced nephrotoxicity in future studies.

In addition, AAs preferentially target PTECs in the kidney because of the selective reabsorption of AAs via organic anion transporters (OATs) [42]. The mitochondrial enrichment and oxygen consumption of the kidney are second only to the heart [43]. Our data also indicated that AAs target to multiple mitochondrial proteins and impair its biological functions. Indeed, AAs could lower MMP and reduce mitochondrial respiration chain and ATP production via JC-1 staining and seahorse experiments. It has been previously reported that AAs induce mitochondrial polarization and inhibit ATP production, thereby leading to apoptosis [28]. Taken together, AAs can induce mitochondrial apoptosis via targeting mitochondrial proteins and impairing their homeostasis.

\section{Conclusions}

In summary, for the first time, activity-based protein profiling (ABPP) in combination with bio-orthogonal click chemistry reaction and cellular thermal shift assay (CETSA), as well metabolomic studies revealed that AAI directly targeting multiple key enzymes in the metabolic process such as lipid metabolism, amino acid metabolism, aerobic respiration, and TCA cycle to impair mitochondrial dysfunction and induce apoptosis in the kidney. Overall, our studies provide novel insight into underlying AAs-induced kidney toxicity and pathogenesis of AAN, which is critical to developing therapeutic strategies for AAN.

\section{Materials and Methods}

\section{Synthesis of aristolochic acid probe}

All the reagents and solvents were purchased from Sigma-Aldrich and AK Scientific, and used without further purification unless stated otherwise.
Reactions were monitored by thin-layer chromatography (TLC). Column chromatography was performed on silica gel $200 \sim 300$ mesh. All ${ }^{1} \mathrm{H}$ NMR (500 MHz), ${ }^{13} \mathrm{C}$ NMR (125 MHz) spectra were recorded. ${ }^{1} \mathrm{H}$ NMR Spectroscopy splitting patterns were designated as singlet $(\mathrm{s})$, doublet $(\mathrm{d})$, triplet $(\mathrm{t})$, quartet (q). Splitting patterns that could not be interpreted or easily visualized were designated as multiplet (m).

A mixture of aristolochic acids $(0.20 \mathrm{mmol})$, $\mathrm{K}_{2} \mathrm{CO}_{3}(41.4 \mathrm{mg}$, $0.30 \mathrm{mmol})$, propargyl bromide, $80 \%$ in toluene $(0.20 \mathrm{mmol})$ in DMF $(2 \mathrm{~mL})$ was stirred at room temperature. Subsequently, the reaction was diluted with $\mathrm{H}_{2} \mathrm{O}(3 \mathrm{~mL})$ and extracted with ethyl acetate $(20 \mathrm{~mL} \times 2)$. The organic phases were combined, washed with brine $(5 \mathrm{~mL} \times 3)$, dried anhydrous $\mathrm{Na}_{2} \mathrm{SO}_{4}$, and concentrated under vacuum. The residue was purified by flash column chromatography, which gave the aristolochic acids probes (AP1 and AP2). ${ }^{1} \mathrm{H}$ NMR, ${ }^{13} \mathrm{C}$ NMR and HRMS of aristolochic acids probes were showed in Supporting information.

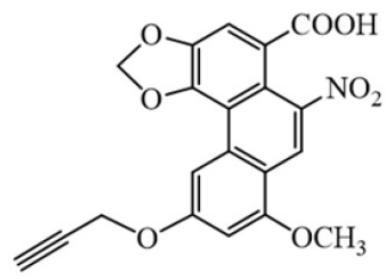

Aristolochic acid probe I (AP1)

Figure A. Yellow solid (yield: 59\%); 'H NMR (500 MHz, DMSO-d 6$) \delta 10.82(\mathrm{~s}, 1 \mathrm{H})$, $8.57(\mathrm{~s}, 1 \mathrm{H}), 8.11(\mathrm{~s}, 1 \mathrm{H}), 7.77(\mathrm{~s}, 1 \mathrm{H}), 6.86(\mathrm{~s}, 1 \mathrm{H}), 6.51(\mathrm{~s}, 2 \mathrm{H}), 4.83(\mathrm{~s}, 2 \mathrm{H}), 4.02(\mathrm{~s}$, $3 \mathrm{H}), 3.62(\mathrm{~s}, 1 \mathrm{H}) ;{ }^{13} \mathrm{C}$ NMR $\left(125 \mathrm{MHz}\right.$, DMSO- $\left.d_{6}\right) \delta 166.1,162.3,158.8,147.5,146.1$, 142.3, 132.2, 121.8, 118.4, 117.1, 112.9, 112.6, 104.3, 103.6, 100.4, 78.8, 78.3, 56.7, 53.2; HRMS m/z: [M-H]- Calcd for $\mathrm{C}_{20} \mathrm{H}_{12} \mathrm{NO}_{8}, 394.0568$, Found 394.0563.

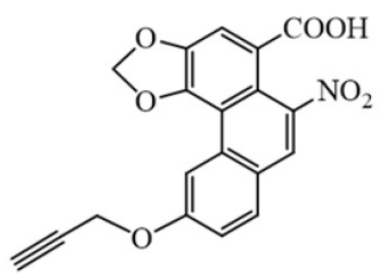

Aristolochic acid probe II (AP2)

Figure B. Yellow solid (yield: 54\%); 'H NMR (500 MHz, DMSO-d $) \delta 10.70(\mathrm{~s}, 1 \mathrm{H})$, $8.59(\mathrm{~s}, 1 \mathrm{H}), 8.49(\mathrm{~s}, 1 \mathrm{H}), 8.13(\mathrm{~d}, J=12 \mathrm{~Hz}, 1 \mathrm{H}), 7.77(\mathrm{~s}, 1 \mathrm{H}), 7.31(\mathrm{~d}, J=12 \mathrm{~Hz}, 1 \mathrm{H})$, $6.53(\mathrm{~s}, 2 \mathrm{H}), 4.84(\mathrm{~s}, 2 \mathrm{H}), 3.63(\mathrm{~s}, 1 \mathrm{H})$; ${ }^{13} \mathrm{C}$ NMR $(125 \mathrm{MHz}$, DMSO-d 6 ) $\delta 166.1,160.6$, $147.4,146.1,143.1,133.2,131.5,127.9,122.0,121.8,119.5,117.9,117.1,112.6$, 111.6, 103.6, 78.8, 78.3, 53.2; HRMS m/z: [M-H] Calcd for $\mathrm{C}_{19} \mathrm{H}_{10} \mathrm{NO}_{7}, 364.0463$, Found 364.0450 .

\section{Cell culture and viability}

Human renal tubular epithelial cell line (HK-2) was obtained from American type culture collection and grown in DMEM/F12 medium (supplemented $10 \%$ fetal bovine serum and $1 \%$ penicillin/ streptomycin), and cultured at $37{ }^{\circ} \mathrm{C}$ and $5 \% \mathrm{CO}_{2}$ in a 
humidified incubator. HK-2 cells were cultured in a 96-well plate for $24 \mathrm{~h}$ and treated with different concentrations of aristolochic acid I (AAI) sodium salt, its analogues (AAIVa and AAIIIa) and AA-probes (AP1 and AP2) for $48 \mathrm{~h}$. The CCK-8 kit was used to detect cell viability.

\section{Animal experiments}

Animal experiments were approved by the Care and Use of Laboratory Animals Center of Shenzhen People's Hospital. C57 BL/ 6 wild-type mice (male, 20 $\pm 2 \mathrm{~g}$, 7-8 weeks old) were treated with aristolochic acid I ( $2 \mathrm{mg} / \mathrm{kg}$, once a day for 4 weeks) by intraperitoneal injection (i.p.). In the control group, mice were administrated with normal saline buffer. After 4 weeks, all mice were anesthetized to collect blood and kidney.

\section{Blood biochemical analysis}

Serum creatinine (CRE) and urea (UREA) were determined by an automatic biochemistry analyzer (TOSHIBA, Japan). Blood routine test was measured by an automatic blood analyzer according to the manufacturer's protocols.

\section{Renal histological analysis}

Kidney tissues were embedded in paraffin and cut into $4 \mu \mathrm{m}$ sections for hematoxylin-eosin (H\&E) staining to evaluate changes in histological morphology.

\section{Enzyme activity assay}

The biological activities of ATP synthase (Abcam, UK) and pyruvate kinase M 1/2 (PKM) (Abcam, UK) were monitored according to the manufacturer's protocols of kits.

\section{Mitochondrial function and cellular oxygen consumption}

The mitochondrial membrane potential (MMP) was determined by using MitoScreen (JC-1 staining) kit (BD biosciences, USA). According to the red/green fluorescence intensity ratio, MMP was evaluated in HK-2 cells. In addition, cellular respiration and mitochondrial function were detected by an oxygen consumption rate (OCR) experiment by using a Seahorse Extracellular Flux Analyzer (Seahorse Bioscience, USA).

\section{Western blot assay}

Crude proteins were extracted from HK-2 cells treated with AAI. Western blot assay was carried out as previously reported [20]. The primary antibodies were used anti-Caspase 3 (Proteintech, USA), anti-Bax (Proteintech, USA), anti-Bcl-2 (Proteintech, USA), anti-Cyt C (Proteintech, USA) and anti- $\beta$-actin
(Affinity biosciences, China). The protein bands were quantified by ImageJ software, and normalized to the corresponding to the $\beta$-actin level.

\section{Fluorescence imaging}

To track the cellular location of the AA-probe, fluorescence imaging studies were performed. HK-2 cells were seeded in the 4-chamber dishes and incubated with AA-probe at different concentrations with or without original compounds. After incubation for $4 \mathrm{~h}$, fluorescence imaging was implemented as previously described [44]. For co-localization of AA-probe and target proteins, immunofluorescence staining was carried out based on probe imaging in situ. The primary antibodies were anti-IDH2 (Abcam, UK), anti-ATP synthase (Abcam, UK), anti-MDH2 (Abcam, UK) and anti-PKM (Proteintech, USA). Finally, cellular imaging was acquired with a confocal fluorescence microscopy (Leica TCS-SP8-SR, Germany).

\section{Fluorescence labeling experiments}

For labeling protein in situ and ex vivo, a fluorescence labeling experiment was performed as previously reported [21, 45]. HK-2 cells in situ were treated with AA-probes in the absence or presence of competitors, proteins were extracted. For the lysate of the kidney ex vivo, proteins first extracted were incubated with probes or competitors. Samples were clicked with a fluorescence dye. Equal amounts of samples were separated by SDS-PAGE gel electrophoresis and analyzed by a fluorescence imaging scanner (Azure Sapphire RGBNIR, USA).

For labeling protein in vivo, the kidneys of mice were collected after the tail vein injection with AP2. Then proteins extracted from the kidney were clicked with a fluorescent dye. Next, experimental procedures were as above described.

\section{Target identification based on LC-MS/MS}

Pull-down and LC-MS/MS experiments were used to identify target proteins of AA. As above described, HK-2 cells and kidney lysates first were incubated with probe or DMSO for $4 \mathrm{~h}$. Protein samples were conjugated with biotin-azide by a clicked reaction. Subsequently, samples were incubated with beads, eluted, reduced, alkylated, and trypsinized to form peptides that were desalted [20]. Ultimately, peptides were labelled by using TMT 10 plex Mass Tag reagents (Thermo, USA) and analyzed by LC-MS/MS (Orbitrap Fusion Lumos, Thermo, USA).

For pull down-western blot assay, the target proteins were validated by western blot assay, procedures were as previously described [20]. 


\section{Target protein data analysis}

According to the TMT protein intensity of DMSO (control) group and AP2 (probe) group. 912 identified target proteins in mice kidney lysis solution were converted into being 860 targets of human homologous genes for further analysis [46]. The differential target proteins were selected basis of the absolute fold change $>1.2$ and $P$ value $<0.05$. Selected proteins were subjected to the heatmap and Volcano plots analysis. Moreover, Veen and GO enrichment analyses were performed on the selected proteins.

\section{Metabolomic analysis by LC-MS/MS}

For the extraction of metabolites in kidney tissue and serum, sample preparation was as previously described [47, 48]. Subsequently, both kidney and serum samples were filtered and then analyzed by LC-MS/MS.

For data analysis, the original file was processed using the Compound Discoverer 3.1 software to perform peak alignment, peak picking, and quantitation for each metabolite by mzCloud, mzVault and MassList database. Metabolite statistical analyses were performed using the MetaboAnalyst 5.0 web servers and statistical software $R$ ( $R$ version R-4.1.1). In chemometrics analysis, Principal components analysis (PCA) and Orthogonal Partial Least Squares-Discriminant Analysis (orthoPLS-DA) were used to discriminate the sample distribution tendency between AAI treatment and control groups. Volcano plots were used to highlight the significant differential metabolites based on $\log 2(\mathrm{FC})$ and $-\log 10(\mathrm{FDR})$ and VIP value of metabolites by ggplot2 in $\mathrm{R}$ language. The significant differential metabolites which have HMDB classification annotation were used to over representation analysis. The metabolic pathways enrichment of differential metabolites was performed using the KEGG database.

\section{Cellular Thermal Shift Assay (CETSA)}

Cellular thermal shift assay (CETSA)-western blot (WB) assay [49] was carried out to further validate AAs' binding to target proteins. Proteins were extracted from HK-2 cells, incubated with AA or DMSO, and then heated by CETSA heat pulse procedure (Applied biosystems, Thermo, USA). Samples were detected by western blot.

\section{Molecular docking analysis}

The 3D structure file of AAI was downloaded from PubChem. The structures of IDH2 (PDB: 1AI2), MDH2 (PDB: 4WLF), PKM (PDB: 4GLN), LDHA (PDB: 5W8J), HK2 (PDB: 2NZT), VDAC1 (PDB: 6G6U), ATP synthase (PDB: 5TSJ) and FASN (PDB: 2PX6) were obtained from RCSB PDB. The AutoDock
Tool was used for preparing structures of the ligand and protein. Subsequently, the AutoDock Vina was employed for docking AAI to these proteins. Pyrx- 0.8 was utilized for molecular docking. All docking results were analyzed and visualized by Pymol.

\section{Statistical analysis}

All data were described as mean \pm SEM values for at least 3 independent experiments. Experimental statistical analysis was performed by one-way ANOVA followed by the Tukey's test in multiple groups. An unpaired two-tailed t-test was carried out in two groups. All statistical analysis was calculated in GraphPad Prism 8.0 software.

\section{Abbreviations}

AA: aristolochic acid; AAN: aristolochic acid nephropathy; ABPP: activity-based protein profiling; CETSA: cellular thermal shift assay; OCR: oxygen consumption rate; $\mathrm{CHN}$ : Chinese herb nephropathy; BEN: Balkan endemic nephropathy; UTUC: upper tract urothelial cancer; TCM: traditional Chinese medicine; PTEC: proximal tubular epithelial cells; MMP: mitochondrial membrane potential; GO: gene ontology; TCA: tricarboxylic acid; PPI: protein and protein interaction; IDH2: isocitrate dehydrogenase 2; MDH2: malate dehydrogenase 2; PKM: pyruvate kinase M1/2; LDH: lactate dehydrogenase A/B; PC: pyruvate carboxylase; VDAC1: voltage-dependent anion-selective channel protein 1; ACACA: acetylCoA carboxylase 1; FASN: fatty acid synthase; HK2: hexokinase-2; CRE: creatinine; QC: quality control; PCA: principal component analysis; Ortho PLS-DA: Orthogonal partial least squares-discriminant analysis; HMDB: human metabolome database; Cyt C: cytochrome C; OATs: organic anion transporters.

\section{Supplementary Material}

Supplementary figures. https://www.ijbs.com/v18p2003s1.pdf

\section{Acknowledgments}

Authors gratefully acknowledge financial support from the National Key Research and Development Program of China (2020YFA0908000); the Innovation Team and Talents Cultivation Program of National Administration of Traditional Chinese Medicine (No: ZYYCXTD-C-202002); the National Natural Science Foundation of China (82074098 and 81903588); the CACMS Innovation Fund (CI2021A05101); the Fundamental Research Funds for the Central public welfare research institutes (ZZ14YQ-050, ZZ14-YQ-051, ZZ14-ND-010, ZZ15-ND-10, ZZ14-FL-002 and ZZ15-YQ-063). 


\section{Author Contributions}

Jigang Wang designed the experiments and supervised the project. Qian Zhang and Piao Luo was responsible for the main experiments, manuscript writing, and data analysis. Jiayun Chen and Chuanbin Yang performed MS and metabolomic data analysis and some experiments in vivo and in vitro, and revised the manuscript. Fei Xia performed probe synthesis. Junzhe Zhang and Huan Tang performed MS experiments. Dandan Liu, Liwei Gu and Xueling He carried out western blot and revised the manuscript. Qiaoli Shi and Tong Yang conducted experimental data and molecular docking analysis.

\section{Ethical statement}

Animal experiments were reviewed and approved by the Ethics Committee of Laboratory Animal Center of Shenzhen People's Hospital, China. All the animal experiments complied with relevant guidelines of the Chinese government and regulations for the care and use of experimental animals.

\section{Competing Interests}

The authors have declared that no competing interest exists.

\section{References}

1. Debelle FD, Vanherweghem JL, Nortier JL. Aristolochic acid nephropathy: a worldwide problem. Kidney Int. 2008; 74: 158-69.

2. Michl J, Ingrouille MJ, Simmonds MS, Heinrich M. Naturally occurring aristolochic acid analogues and their toxicities. Nat Prod Rep. 2014; 31: 676-93.

3. Kumar V, Poonam, Prasad AK, Parmar VS. Naturally occurring aristolactams, aristolochic acids and dioxoaporphines and their biological activities. Nat Prod Rep. 2003; 20: 565-83

4. Xu T, Chen W, Zhou J, Dai J, Li Y, Zhao Y. Computational Analysis of Naturally Occurring Aristolochic Acid Analogues and Their Biological Sources. Biomolecules. 2021; 11.

5. Final report on carcinogens background document for aristolochic acids. Rep Carcinog Backgr Doc. 2008: i-xxv, 1-246.

6. Pavlović NM, Maksimović V, Maksimović JD, Orem WH, Tatu CA, Lerch HE et al. Possible health impacts of naturally occurring uptake of aristolochic acids by maize and cucumber roots: links to the etiology of endemic (Balkan) nephropathy. Environ Geochem Health. 2013; 35: 215-26.

7. Jelaković B, Dika Ž, Arlt VM, Stiborova M, Pavlović NM, Nikolić J, et al. Balkan Endemic Nephropathy and the Causative Role of Aristolochic Acid. Semin Nephrol. 2019; 39: 284-96.

8. Gruia AT, Oprean C, Ivan A, Cean A, Cristea M, Draghia L, et al. Balkan endemic nephropathy and aristolochic acid I: an investigation into the role of soil and soil organic matter contamination, as a potential natural exposure pathway. 2018; 40: 1437-48.

9. Michl J, Jennings HM, Kite GC, Ingrouille MJ, Simmonds MS, Heinrich M. Is aristolochic acid nephropathy a widespread problem in developing countries? A case study of Aristolochia indica L. in Bangladesh using an ethnobotanical-phytochemical approach. J Ethnopharmacol. 2013; 149: 235-44.

10. Poon SL, Pang ST, McPherson JR, Yu W, Huang KK, Guan P, et al. Genome-wide mutational signatures of aristolochic acid and its application as a screening tool. Sci Transl Med. 2013; 5: 197ra01.

11. Ng AWT, Poon SL, Huang MN, Lim JQ, Boot A, Yu W, et al. Aristolochic acids and their derivatives are widely implicated in liver cancers in Taiwan and throughout Asia. Sci Transl Med. 2017; 9: eaan6446.

12. Bastek H, Zubel T, Stemmer K, Mangerich A, Beneke S, Dietrich DR Comparison of Aristolochic acid I derived DNA adduct levels in human renal toxicity models. Toxicology. 2019; 420: 29-38.

13. Prabu SM, Muthumani M. Silibinin ameliorates arsenic induced nephrotoxicity by abrogation of oxidative stress, inflammation and apoptosis in rats. Mol Biol Rep. 2012; 39: 11201-16.

14. Pozdzik AA, Salmon IJ, Husson CP, Decaestecker C, Rogier E, Bourgeade MF, et al. Patterns of interstitial inflammation during the evolution of renal injury in experimental aristolochic acid nephropathy. Nephrol Dial Transplant. 2008; 23: $2480-91$
15. Hsin $\mathrm{YH}$, Cheng CH, Tzen JT, Wu MJ, Shu KH, Chen HC. Effect of aristolochic acid on intracellular calcium concentration and its links with apoptosis in renal tubular cells. Apoptosis. 2006; 11: 2167-77.

16. Zhu S, Wang Y, Jin J, Guan C, Li M, Xi C, et al. Endoplasmic reticulum stress mediates aristolochic acid I-induced apoptosis in human renal proximal tubular epithelial cells. Toxicol In vitro. 2012; 26: 663-71.

17. Jadot I, Declèves AE, Nortier J, Caron N. An Integrated View of Aristolochic Acid Nephropathy: Update of the Literature. Int J Mol Sci. 2017; 18.

18. Pfau W, Schmeiser HH, Wiessler M. Aristolochic acid binds covalently to the exocyclic amino group of purine nucleotides in DNA. Carcinogenesis. 1990; 11: 313-9.

19. Randerath K, Reddy MV, Gupta RC. 32P-labeling test for DNA damage. Proc Natl Acad Sci U S A. 1981; 78: 6126-9.

20. Liu DD, Luo P, Gu L, Zhang $\mathrm{Q}$, Gao P, Zhu Y, et al. Celastrol exerts a neuroprotective effect by directly binding to HMGB1 protein in cerebral ischemia-reperfusion. Journal of Neuroinflammation. 2021; 18: 174.

21. Wang J, Zhang CJ, Chia WN, Loh CC, Li Z, Lee YM, et al. Haem-activated promiscuous targeting of artemisinin in Plasmodium falciparum. Nat Commun. 2015; 6: 10111

22. Wang J, Zhang J, Zhang CJ, Wong YK, Lim TK, Hua ZC, et al. In situ Proteomic Profiling of Curcumin Targets in HCT116 Colon Cancer Cell Line. Sci Rep. 2016; 6: 22146

23. Wang J, Tan XF, Nguyen VS, Yang P, Zhou J, Gao M, et al. A quantitative chemical proteomics approach to profile the specific cellular targets of andrographolide, a promising anticancer agent that suppresses tumor metastasis. Mol Cell Proteomics. 2014; 13: 876-86.

24. Jafari R, Almqvist $H$, Axelsson $H$, Ignatushchenko M, Lundbäck T, Nordlund $\mathrm{P}$, et al. The cellular thermal shift assay for evaluating drug target interactions in cells. Nat Protoc. 2014; 9: 2100-22.

25. Clark AJ, Parikh SM. Mitochondrial Metabolism in Acute Kidney Injury. Semin Nephrol. 2020; 40: 101-13.

26. Emma F, Montini G, Parikh SM, Salviati L. Mitochondrial dysfunction in inherited renal disease and acute kidney injury. Nat Rev Nephrol. 2016; 12: 267-80.

27. Deng HF, Yue LX, Wang NN, Zhou YQ, Zhou W, Liu X, et al. Mitochondrial Iron Overload-Mediated Inhibition of Nrf2-HO-1/GPX4 Assisted ALI-Induced Nephrotoxicity. Front Pharmacol. 2020; 11: 624529.

28. Liu X, Wu J, Wang J, Feng X, Wu H, Huang R, et al. Mitochondrial dysfunction is involved in aristolochic acid I-induced apoptosis in renal proximal tubular epithelial cells. Hum Exp Toxicol. 2020; 39: 673-82.

29. Luciano RL, Perazella MA. Aristolochic acid nephropathy: epidemiology, clinical presentation, and treatment. Drug Saf. 2015; 38: 55-64.

30. Hoang ML, Chen CH, Chen PC, Roberts NJ, Dickman KG, Yun BH, et al. Aristolochic Acid in the Etiology of Renal Cell Carcinoma. Cancer Epidemiol Biomarkers Prev. 2016; 25: 1600-8.

31. Lu ZN, Luo Q, Zhao LN, Shi Y, Wang N, Wang L, et al. The Mutational Features of Aristolochic Acid-Induced Mouse and Human Liver Cancers. Hepatology. 2020; 71: 929-42.

32. Stiborová M, Bárta F, Levová $\mathrm{K}$, Hodek $\mathrm{P}$, Schmeiser $\mathrm{HH}$, Arlt VM, et al. A Mechanism of O-Demethylation of Aristolochic Acid I by Cytochromes P450 and Their Contributions to This Reaction in Human and Rat Livers: Experimental and Theoretical Approaches. Int J Mol Sci. 2015; 16: 27561-75.

33. Stiborová M, Arlt VM, Schmeiser HH. DNA Adducts Formed by Aristolochic Acid Are Unique Biomarkers of Exposure and Explain the Initiation Phase of Upper Urothelial Cancer. Int J Mol Sci. 2017; 18: 2144.

34. Wang Z, He B, Liu Y, Huo M, Fu W, Yang C, et al. In situ metabolomics in nephrotoxicity of aristolochic acids based on air flow-assisted desorption electrospray ionization mass spectrometry imaging. Acta Pharm Sin B. 2020; 10: 1083-93

35. Li Y, Xu H, Cai D, Zhu S, Liu X, Zhao Y, et al. Integration of transcriptomic, proteomic and metabolomic data to reveal the biological mechanisms of AAI injury in renal epithelial cells. Toxicol In vitro. 2021; 70: 105054

36. Michl J, Bello O, Kite GC, Simmonds MSJ, Heinrich M. Medicinally Used Asarum Species: High-Resolution LC-MS Analysis of Aristolochic Acid Analogs and In vitro Toxicity Screening in HK-2 Cells. Front Pharmacol. 2017; 8. 215 .

37. Cui Y, Han J, Ren J, Chen H, Xu B, Song N, et al. Untargeted LC-MS-based metabonomics revealed that aristolochic acid I induces testicular toxicity by inhibiting amino acids metabolism, glucose metabolism, $\beta$-oxidation of fatty acids and the TCA cycle in male mice. Toxicol Appl Pharmacol. 2019; 373: 26-38.

38. Cascón A, Comino-Méndez I, Currás-Freixes M, de Cubas AA, Contreras L Richter S, et al. Whole-exome sequencing identifies $\mathrm{MDH} 2$ as a new familial paraganglioma gene. J Natl Cancer Inst. 2015; 107.

39. Brandi J, Cecconi D, Cordani M, Torrens-Mas M, Pacchiana R, Dalla Pozza E, et al. The antioxidant uncoupling protein 2 stimulates hnRNPA2/B1, GLUT1 and PKM2 expression and sensitizes pancreas cancer cells to glycolysis inhibition. Free Radic Biol Med. 2016; 101: 305-16.

40. Puig T, Turrado C, Benhamú B, Aguilar H, Relat J, Ortega-Gutiérrez S, et al. Novel Inhibitors of Fatty Acid Synthase with Anticancer Activity. Clin Cancer Res. 2009; 15: 7608-15.

41. Lee HJ, Li CF, Ruan D, He J, Montal ED, Lorenz S, et al. Non-proteolytic ubiquitination of Hexokinase 2 by HectH9 controls tumor metabolism and cancer stem cell expansion. Nat Commun. 2019; 10: 2625. 
42. Zeng Y, Zhang R, Wu J, Liu M, Peng W, Yu X, et al. Organic anion transporter 1 (OAT1) involved in renal cell transport of aristolochic acid I. Hum Exp Toxicol. 2012; 31: 759-70

43. Bhargava P, Schnellmann RG. Mitochondrial energetics in the kidney. Nat Rev Nephrol. 2017; 13: 629-46

44. Cheng K, Lee JS, Hao P, Yao SQ, Ding K, Li Z. Tetrazole-Based Probes for Integrated Phenotypic Screening, Affinity-Based Proteome Profiling, and Sensitive Detection of a Cancer Biomarker. Angew Chem Int Ed Engl. 2017; 56: 15044-8.

45. Ma N, Hu J, Zhang ZM, Liu W, Huang M, Fan Y, et al. 2H-Azirine-Based Reagents for Chemoselective Bioconjugation at Carboxyl Residues Inside Live Cells. J Am Chem Soc. 2020; 142: 6051-9.

46. Smedley D, Haider S, Ballester B, Holland R, London D, Thorisson G, et al. BioMart--biological queries made easy. BMC Genomics. 2009; 10: 22.

47. Want EJ, Masson P, Michopoulos F, Wilson ID, Theodoridis G, Plumb RS, et al. Global metabolic profiling of animal and human tissues via UPLC-MS. Nat Protoc. 2013; 8: 17-32.

48. Barri T, Dragsted LO. UPLC-ESI-QTOF/MS and multivariate data analysis for blood plasma and serum metabolomics: effect of experimental artefacts and anticoagulant. Anal Chim Acta. 2013; 768: 118-28.

49. Dai L, Prabhu N, Yu LY, Bacanu S, Ramos AD, Nordlund P. Horizontal Cell Biology: Monitoring Global Changes of Protein Interaction States with the Proteome-Wide Cellular Thermal Shift Assay (CETSA). Annu Rev Biochem. 2019; 88: 383-408. 